\title{
Emotion experience and frailty in a sample of Italian community-dwelling older adults
}

This article was published in the following Dove Press journal:

Clinical Interventions in Aging

\author{
Anna Mulasso ${ }^{1,2}$ \\ Laura Argiolu' \\ Mattia Roppolo' \\ Danny Azucar' \\ Emanuela Rabaglietti'
}

'Department of Psychology, University of Turin, Turin, Italy; ${ }^{2}$ NeuroMuscular

Function Research Group, School of Exercise and Sport Sciences,

Department of Medical Sciences, University of Turin, Turin, Italy
Correspondence: Mattia Roppolo

Department of Psychology, University of Turin, Via Verdi, I0, I0I24 - Turin, Italy

Tel +390 II 6702788

Fax +39 0116702791

Email mattia.roppolo@unito.it

\begin{abstract}
Frailty increases individual vulnerability to external stressors and involves high risk for adverse geriatric outcomes. To date, few studies have addressed the role of emotion perception and its association with frailty in aged populations. This cross-sectional study aimed to explore whether a significant association between frailty and emotional experience exists in a sample of Italian community-dwelling older adults. Our sample consisted of 104 older adults (age $76 \pm 8$ years; $59.6 \%$ women) living in Piedmont, Italy. Frailty was measured using the Italian version of the Tilburg Frailty Indicator (TFI), and emotion perception was measured with the Positive and Negative Affect Schedule (PANAS). The Mini-Mental State Examination was used as a screening tool for cognitive functions (people with a score $\leq 20$ points were excluded). One-way analysis of covariance (ANCOVA), adjusted for interesting variables, and post hoc tests were performed where appropriate. According to the TFI, $57.7 \%$ of participants resulted as frail. Analysis showed a significant greater severity of frailty in the low positive affect (PA) group compared to the high PA group. Similarly, those with high negative affect (NA) showed significantly higher levels of frailty than the low NA group. As expected, significant differences for frailty were also found among the groups composed of 1) people with high PA and low NA, 2) people with low PA or high NA, and 3) people with low PA and high NA. Post hoc tests showed a greater severity of frailty in the second and in the third groups compared to the first one. Lastly, robust participants aged $>75$ years showed higher levels of PA than the group aged between 60 and 75 years. These findings demonstrate that both PA and NA may influence frailty, giving new insights for the evaluation and prevention of frailty in older adults.
\end{abstract}

Keywords: emotion perception functional decline, frail individuals, Tilburg Frailty Indicator, analysis of covariance

\section{Introduction}

In affective and emotional experience, it is possible to recognize positive affect (PA) and negative affect (NA). The first reflects the condition in which the individual feels enthusiastic, active, excited, and determined; the second is a general dimension of subjective distress, including contempt, anger, disgust, guilt, fear, shame, and so on. ${ }^{1}$ Tellenger ${ }^{2}$ demonstrated that PA and NA correspond to extraversion personality factors and anxiety/neuroticism, respectively. The presence of positive emotions is a factor related to individual health and well-being, especially among older people. PA is strictly related to engagement in social activity, higher self-reported frequency of positive events in a person's life, higher perception of satisfaction, and good physical health. ${ }^{3-6}$ Furthermore, studies conducted in older adults found that PA was associated with lower disability in the execution of daily living activities, ${ }^{7}$ higher levels of mobility, good functional status, and major likelihood of survival at 2 years, ${ }^{8}$ as well as higher rate of recovery of functional status after a stroke. ${ }^{9}$ On the other hand, 
NA is correlated with stress sensations and poor coping abilities. $^{10}$

Furthermore, according to the socioemotional selectivity theory (SST), ${ }^{11}$ emotional experience improves with age because people come to appreciate and invest more effort in matters of life that are important to them. In other words, interpersonal relationships in older people are aimed at achieving emotional satisfaction and are carefully selected. The result of this behavior is that older people, being more selective in relationships, have more positive emotions. ${ }^{12}$ Charles et al, ${ }^{13}$ when comparing groups of different ages, found that older adults have a stronger capacity to remember positive images and they were more inclined to process and remember positive information, with the resulting emotional content. This trend has been described by Carstensen and Mikels as a "positivity effect." 14

Regarding age-related problems, frailty is one of the most widely spread and disabling conditions. Frailty was present in $12.7 \%-44.6 \%$ of Italian older adults, depending on the index used. ${ }^{15}$ It increases individual vulnerability to external stressors and consequently increases certain negative health outcomes. ${ }^{16,17}$ Gobbens et al defined frailty as "a dynamic state affecting an individual who experiences losses in one or more domains of human functioning (physical, psychological, social)" on the basis of a biopsychosocial model. ${ }^{18}$ This view of frailty has recently gained more consensus as it conceives frailty as a multidimensional condition..$^{19,20}$ Gobbens et a ${ }^{18}$ believe that frailty affects physical, psychological, and social domains of human functioning.

However, the role of affectivity in relation to frailty has not been widely investigated in the literature. To the best of our knowledge, only two studies demonstrated that PA reduced the risk for frailty in older adults who were not frail at baseline. ${ }^{21,22}$ However, those authors used a physical conceptualization of frailty, excluding psychological and social components of frailty, and they analyzed exclusively the role of PA in relationship with frailty, evaluating PA with four positive items of the Center of Epidemiologic Study Depression Scale. Within this picture, there is a need to learn more about the association of affectivity and frailty, considering affectivity as combination of PA and NA, and frailty as a multidimensional construct. The first hypothesis of our study is that PA and NA may affect frailty condition, with a greater severity of frailty condition in individuals with low levels of PA or high levels of NA. Our second hypothesis sustains that PA, on the basis of SST, increases with the advancement of age in robust individuals, while it remains constant, or worsens, in frail individuals. The present study aimed to investigate the existence of a significant association between affectivity and frailty in a sample of Italian communitydwelling older adults. The following specific objectives will be pursued: 1) to evaluate differences in frailty - including physical, psychological, and social domains - among groups of individuals with different levels of PA, NA, and total emotion perception, 2) to test differences in PA between groups of differing ages and on the basis of frailty condition.

\section{Materials and methods Study population and procedures}

This preliminary study, part of a larger research project carried out by the University of Turin, aimed to investigate the living and health conditions of older adults in Piedmont, Italy. The study was approved by the Bioethical Committee of the University of Turin. Participant recruitment was done through direct contact between the project coordinator and the available senior associations $(\mathrm{N}=5)$ located in Piedmont. To explain the aims and to answer any questions about the study, a preliminary meeting was organized with each association. The participants did not receive any reward or compensation for their participation. In accordance with the Italian laws and the Code of Ethics of the American Psychological Association, ${ }^{23}$ participants read and signed the informed consent form that described the aims and the methodology of the research.

In total, 175 people were contacted, of whom 67 (38.3\%) did not wish to participate and 4 (2.3\%) did not meet the inclusion criteria of the study. A final sample of 104 participants (59.4\%) took part in this study. Participants were recruited if they satisfied the following criteria: 1) they were aged $>60$ years, 2) they were able to understand and speak Italian, 3) they had a Mini-Mental State Examination (MMSE) score $\geq 21$ points, ${ }^{24} 4$ ) they lived in their home and not in residential care facilities. Participants were excluded from the study if they had acute health problems in the previous month, such as a stroke, surgical operation, fractures, and so on.

The cognitive test and questionnaires were administered in the senior associations by two trained psychologists. First, the cognitive test was administered, followed by the self-report questionnaires that were filled out autonomously. About 15 minutes was required to complete the questionnaires. If necessary, the psychologist helped the older adults fill out the questionnaires, reading and explaining each item. Data collection was carried out between the months of March and June 2016.

\section{Measures}

Emotions were investigated using the Positive and Negative Affect Schedule (PANAS). ${ }^{25}$ PANAS is a self-report 
questionnaire designed to investigate positive and negative emotions during the past weeks. It is composed of 20 items, 10 of which are positive adjectives and the other 10 are negative adjectives. The positive adjectives refer to positive emotions such as determined, excited, strong, proud, and so on, while the negative adjectives refer to negative emotions such as distressed, frightened, irritable, disturbed, and so on. The individual must evaluate each emotion with a score from 1 (for nothing) to 5 (very) based on how he/she identifies in reference to that emotion in the past few weeks. The factorial structure of the test assumes two factors, which correspond to the two subscales (PA and NA); they are to be considered independent from each other. Therefore, for each subscale, the score can range between 10 and $50 .{ }^{1}$ Reliability and validity of PANAS have been previously tested in aged people..$^{26,27}$ Studies have found a slight correlation between the positive and negative subscales independently from age, but the two dimensions can still be considered independent. ${ }^{27}$ In this study, we used the Italian version of PANAS validated by Terraciano et al. ${ }^{28}$ The coefficient of consistency between the original version and the Italian one was $0.98 .{ }^{28}$ In our study, the PANAS positive and negative scales showed a reliability of 0.90 and 0.88 , respectively. Following the procedure described by Garcia et al, ${ }^{29}$ participants were divided, using the median value of the PA scale, into two groups of similar size, namely, high PA if they obtained a score $>36$ points and low PA if they had a score $\leq 36$ points. Similarly, using the median value of the NA subscale, participants were divided into low NA if they received a score $<17$ and high NA if they received a score $\geq 17$. At the end, a new categorical variable related to total emotion perception was created using the following criteria: the first group included people with high PA and low NA (called self-fulfilling), the second included those with low PA and low NA (defined as low affective) or high PA and high NA (called high affective), and the third included those with low PA and high NA (defined as selfdestructive), on the basis of scientific evidence. ${ }^{29-31}$ In this study, low affective and high affective groups were combined in order to ensure a sufficient sample size for the analysis.

Frailty was measured using the Italian version of the Tilburg Frailty Indicator (TFI). ${ }^{32,33}$ The TFI is a self-report questionnaire composed of two parts: part A consists of 10 questions that investigate the determinants of frailty (gender, age, marital status, level of education, lifestyle, etc) and part B consists of 15 items that investigate frailty belonging to three domains of human functioning: physical, psychological, and social. The physical domain consists of eight questions related to physical activity, unexplained weight loss, difficulty in walking and balancing, hearing and vision problems, loss of strength in hands, and physical tiredness. The psychological domain includes four items about cognition, depressive symptoms, anxiety, and coping. Finally, social domain consists of three questions concerning living alone, social relationships, and perceived social support. Eleven questions from part B have two categories of answer choices: "yes" and "no," while the other includes three: "yes," "sometimes," and "no." All of the items were dichotomized in 0 and 1 . The total score of the TFI is between 0 and 15. Scores range from 0 to 8 for the physical domain, from 0 to 4 for the psychological domain, and from 0 to 3 for the social domain. Higher scores correspond to a more serious frailty status. The cut-off value that distinguishes frail from robust individuals is $5 .^{33}$ A Cronbach's $\alpha$ of 0.72 was obtained in the present study.

Finally, cognitive functioning was evaluated using the MMSE. ${ }^{34}$ The MMSE investigates six mental functioning areas: orientation, recording, attention, re-evocation, language, and constructive praxis. The minimum score for this tool is 0 up to a maximum of 30 points. High scores correspond to better cognitive functions.

\section{Statistical analysis}

Data were processed using the Statistical Package for Social Sciences (SPSS), Version 24 (IBM Corporation, Armonk, NY, USA). Statistical significance level was fixed at $\alpha<0.05$ for all tests. Descriptive statistics were conducted for all the variable of the study. Internal consistency of the scales was carried out using Cronbach's $\alpha$. Values of $\alpha \geq 0.70$ were considered acceptable. ${ }^{35}$

In order to determine whether there were statistically significant differences in frailty (total frailty, physical frailty, psychological frailty, and social frailty) between individuals with different levels of emotion perception (high and low PA, high and low NA, and the total emotion perception), an analysis of covariance (ANCOVA), controlling for age and gender of participants, was performed. In total, 12 models based on ANCOVA were analyzed. For each statistically significant effect of the variable total emotion perception, the Bonferroni post hoc test was executed.

Finally, to test whether the positive emotional experience was statistically different between "young" older adults (60-75 years) and older adults ( $\geq 76$ years), an ANCOVA controlling for gender was carried out on the total sample. The same two models based on ANCOVA were tested selecting exclusively robust participants followed by frail participants.

\section{Results}

The sample is composed of 104 older adults, of which $62(59.6 \%)$ are females, with a mean age of 76 years $(\mathrm{SD}=8$; 
range 61-95 years). Most of the participants were married $(49.0 \%)$, were born in the Islands or in the South of Italy $(65.3 \%)$, had a level of education corresponding to primary (34.6\%) or secondary school (32.7\%), and performed manual labor (55.7\%). A large number of participants (52.9\%) reported having one or more chronic disease. The mean MMSE total score was 26.7 ( $\mathrm{SD}=2.6$; range 21-30) points. With respect to frailty, the TFI reported a mean value of 5.36 ( $\mathrm{SD}=2.96$, range $0-12$ ) points, with $57.7 \%$ of individuals in a condition of frailty. Considering the emotion perception, the PANAS-PA scale and the PANAS-NA scale showed mean values of 35.13 ( $\mathrm{SD}=7.43$; range $16-50)$ and 17.95 points ( $\mathrm{SD}=6.96$; range 10-43), respectively. The characteristics of the sample are summarized in Tables 1 and 2 .

The ANCOVA, controlling for gender and age, reported differences in frailty according to levels of emotion perception. Specifically, significant differences were found between the high PA and the low PA groups for multidimensional frailty ( $p=0.007$, adjusted $R^{2}=29.6 \%$ ), physical frailty ( $p=0.008$, adjusted $R^{2}=24.7 \%$ ), and psychological frailty ( $p=0.041$, adjusted $R^{2}=9.6 \%$ ). In all the three models, the

Table I Characteristics of participants $(\mathrm{N}=104)$

\begin{tabular}{|c|c|c|}
\hline Variables & n (\%) & Mean (SD) \\
\hline Age, years & & $76(8)$ \\
\hline Gender, $\mathrm{n}(\%)$ of female & $62(59.6)$ & \\
\hline \multicolumn{3}{|l|}{ Marital status } \\
\hline Married & $5 I(49.0)$ & \\
\hline Not married & $9(8.7)$ & \\
\hline Widowed & $40(38.5)$ & \\
\hline Divorced & $4(3.8)$ & \\
\hline \multicolumn{3}{|l|}{ Level of education } \\
\hline No schooling & $14(13.5)$ & \\
\hline Primary school, 5 years & $36(34.6)$ & \\
\hline Secondary school, 8 years & $34(32.7)$ & \\
\hline High school diploma, 13 years & $13(12.5)$ & \\
\hline University degree, 18 years & $7(6.7)$ & \\
\hline Past job, $\mathrm{n}(\%)$ of manual workers & $58(55.7)$ & \\
\hline MMSE & & $26.7(2.6)$ \\
\hline Chronic disease, $n(\%)$ of yes & $55(52.9)$ & \\
\hline \multicolumn{3}{|l|}{ Lifestyle } \\
\hline Healthy & $54(52.0)$ & \\
\hline More or less healthy & $43(4 \mid .3)$ & \\
\hline Unhealthy & $7(6.7)$ & \\
\hline \multicolumn{3}{|l|}{ Life events, $n(\%)$ of yes } \\
\hline Lost somebody close & $39(37.5)$ & \\
\hline Serious disease & $15(14.4)$ & \\
\hline Serious disease in some close relation & $37(35.6)$ & \\
\hline End of important relationship & $2(1.9)$ & \\
\hline Traffic accident & $4(3.8)$ & \\
\hline Crime & $9(8.7)$ & \\
\hline Satisfaction of housing environment, $n(\%)$ of yes & $91(87.5)$ & \\
\hline
\end{tabular}

Note: Higher scores of MMSE corresponded to a better cognitive status. Abbreviations: SD, standard deviation; MMSE, Mini-Mental State Examination.
Table 2 Characteristics of participants on components of frailty and emotion perception ( $\mathrm{N}=104)$

\begin{tabular}{lll}
\hline Variables & $\mathbf{n}(\%)$ & Mean (SD) \\
\hline TFI & & $5.36(2.96)$ \\
Physical TFI & & $2.66(1.94)$ \\
Psychological TFI & $1.66(0.99)$ \\
Social TFI & & $1.03(0.95)$ \\
Level of frailty* & \\
Frail persons & $60(57.7)$ & \\
Robust persons & $44(42.3)$ & $35.13(7.43)$ \\
PANAS positive & & $17.95(6.96)$ \\
PANAS negative & & \\
\hline
\end{tabular}

Notes: *Individuals with a score $\geq 5$ were classified as frail. Higher TFI scores corresponded to severe frailty condition. Higher scores of the positive and the negative subscales of PANAS indicated higher perception of positive and negative emotion perceptions, respectively.

Abbreviations: SD, standard deviation; TFI, Tilburg Frailty Indicator; PANAS, Positive and Negative Affect Schedule.

low PA group showed a significant greater severity of frailty condition in comparison with the high PA group. No differences were found between high PA and low PA groups for social frailty ( $p>0.05$ ) (Table 3 , first five columns).

Similarly, the ANCOVA, adjusted for the same previous variables, revealed significant differences between the low NA and the high NA groups in terms of multidimensional frailty $\left(p<0.001\right.$, adjusted $\left.R^{2}=35.6 \%\right)$, physical frailty ( $p=0.001$, adjusted $R^{2}=27.5 \%$ ), and psychological frailty $\left(p<0.001\right.$, adjusted $\left.R^{2}=19.6 \%\right)$. In all the three models, the high NA group showed higher scores of frailty compared to the low NA group. There were no statistical differences between low NA and high NA groups for social frailty $(p>0.05)$ (Table 3, last four columns).

Lastly, the ANCOVA analysis, controlled for the same previous variables, demonstrated the existence of significant differences among the three groups composed of 1) people with high PA and low NA, 2) people with low PA and low NA or high PA and high NA, and 3) people with low PA and high NA, for multidimensional frailty ( $p<0.001$, adjusted $\left.R^{2}=38.7 \%\right)$, physical frailty $(p<0.001$, adjusted $\left.R^{2}=31.5 \%\right)$, and psychological frailty $(p<0.001$, adjusted $R^{2}=19.9 \%$ ). No differences were detected among the three groups for social frailty $(p>0.05)$. Specifically, post hoc tests revealed significant differences in terms of multidimensional, physical, and psychological frailty between the first (high PA and low NA) and the second (low PA and low NA or high PA and high NA) groups, as well as between the first (high PA and low NA) and the third (low PA and high NA) groups, with a greater severity of frailty condition in the second and in the third groups compared to the first one. No differences were 
Table 3 Frailty condition based on positive and negative affect

\begin{tabular}{|c|c|c|c|c|c|c|c|c|}
\hline \multirow[t]{2}{*}{ Variables } & \multicolumn{4}{|l|}{ Positive emotions } & \multicolumn{4}{|c|}{ Negative emotions } \\
\hline & High PA $(n=52)$ & Low PA $(n=52)$ & $F(1,100)$ & $p$-value* & Low NA (n=5 I) & High NA $(n=53)$ & $F(1,100)$ & $p$-value* \\
\hline Multidimensional frailty & $4.25 \pm 2.76$ & $6.46 \pm 2.76$ & 7.64 & 0.007 & $4.06 \pm 2.80$ & $6.60 \pm 2.96$ & 17.76 & $<0.001$ \\
\hline Physical frailty & $1.98 \pm 1.64$ & $3.35 \pm 2.00$ & 7.42 & 0.008 & $1.94 \pm 1.84$ & $3.36 \pm 1.80$ & 11.56 & 0.001 \\
\hline Psychological frailty & $1.38 \pm 1.09$ & $1.94 \pm 0.80$ & 4.31 & $0.04 I$ & $1.24 \pm 0.95$ & $2.08 \pm 0.85$ & 17.25 & $<0.001$ \\
\hline Social frailty & $0.88 \pm 0.92$ & I. $17 \pm 0.96$ & 0.12 & NS & $0.88 \pm 0.89$ & $1.17 \pm 1.00$ & 0.59 & NS \\
\hline
\end{tabular}

Notes: *Values based on ANCOVA, using gender and age of participants as covariates. Data represent mean \pm SD.

Abbreviations: ANCOVA, analysis of covariance; high PA, high positive affect (score of PANAS-Positive subscale $>36$ points); low PA, low positive affect (score of PANAS-Positive subscale $\leq 36$ points); low NA, low negative affect (score of PANAS-Negative subscale $<17$ points); high NA, high negative affect (score of PANAS-Negative subscale $\geq 17$ points); NS, nonsignificant.

found between the second (low PA and low NA or high PA and high NA) and the third (high PA and low NA) groups (Table 4).

Another model based on ANCOVA, adjusted for gender, was used to test the differences in positive emotion perception according to age groups. The model showed that there are no significant differences between "young" older adults (60-75 years) and older adults ( $>75$ years) for perception of PA ( $p>0.05)$, when both robust and frail individuals were considered at the same time $(\mathrm{N}=104)$. On the contrary, when the same analysis was conducted on the group of robust participants $(n=44)$, our results yielded significant differences $(p=0.017)$, with higher levels of PA in the group of adults aged $>75$ years. No significant differences were found in terms of PA if the same model was tested in the group of frail participants $(n=60)$ (Table 5).

\section{Discussion}

This cross-sectional research explored the role of emotion experience on the condition of frailty in a sample of older adults living in Italy. The purpose of this study was twofold. On the one hand, it analyzed the condition of frailty in groups with different levels of PA and NA; frailty was analyzed from a multidimensional, physical, psychological, and social point of view. On the other hand, it tested whether there were differences in terms of PA in groups of individuals of different ages and on the basis of frailty condition.

Data on frailty prevalence obtained in this study are in line with the results of other surveys conducted on Italian aged populations, ${ }^{20,36}$ where pre-frail and frail older adults are larger than robust ones.

First, these findings demonstrate that both PA and NA may affect frailty condition. Specifically, individuals who experienced high PA presented a lower score of multidimensional, physical, and psychological frailty when compared to those who experienced low PA. Furthermore, people with low NA exhibited a reduced level of frailty in comparison with those with high NA. Taking into consideration the total emotion perception, results showed that the group who experienced high PA and low NA (self-fulfilling) had a significantly lower severity of multidimensional, physical, and psychological frailty condition in comparison with those who experienced low PA and/or high NA (low affective and high affective, self-destructive). Conversely, in our study, emotion perception did not affect social frailty. Consistent with these results, previous longitudinal studies demonstrated the protective role of PA on frailty. ${ }^{21,22}$ Those authors showed that increasing PA values at baseline, measured using four positive items of the Center of Epidemiologic Study Depression Scale, reduced the risk for physical frailty in older adults. It is possible to

Table 4 Frailty condition based on total emotion perception

\begin{tabular}{|c|c|c|c|c|c|}
\hline \multicolumn{6}{|c|}{ Total emotion perception } \\
\hline Variables & $\begin{array}{l}\text { High PA } \\
\text { and low NA } \\
(n=3 I)\end{array}$ & $\begin{array}{l}\text { Low PA and low NA or } \\
\text { high PA and high NA } \\
(n=4 I)\end{array}$ & $\begin{array}{l}\text { Low PA } \\
\text { and high NA } \\
(n=32)\end{array}$ & $F(2,99)$ & $p$-value \\
\hline Multidimensional frailty & $3.10 \pm 2.34$ & $5.76 \pm 2.63 * *$ & $7.03 \pm 2.58 * *$ & 12.38 & $<0.001$ \\
\hline Physical frailty & $1.35 \pm 1.40$ & $2.88 \pm 1.81 * *$ & $3.66 \pm 1.91 * *$ & 9.50 & $<0.00$ I \\
\hline Psychological frailty & $1.00 \pm 1.00$ & $1.78 \pm 0.88 * *$ & $2.16 \pm 0.77 * *$ & 9.38 & $<0.001$ \\
\hline Social frailty & $0.74 \pm 0.86$ & $1.10 \pm 0.94$ & $1.22 \pm 1.01$ & 0.282 & NS \\
\hline
\end{tabular}

Notes: *Values based on ANCOVA, using gender and age as covariates. The values of multiple comparison were based on Bonferroni post hoc test: $* * p<0.05$ versus high $P A$ and low NA group. Data represent mean \pm SD.

Abbreviations: ANCOVA, analysis of covariance; high PA, high positive affect (score of PANAS-Positive subscale $>36$ points); low PA, low positive affect (score of PANAS-positive subscale $\leq 36$ points); low NA, low negative affect (score of PANAS-Negative subscale $<$ I 7 points); high NA, high negative affect (score of PANAS-Negative subscale $\geq 17$ points); NS, nonsignificant. 


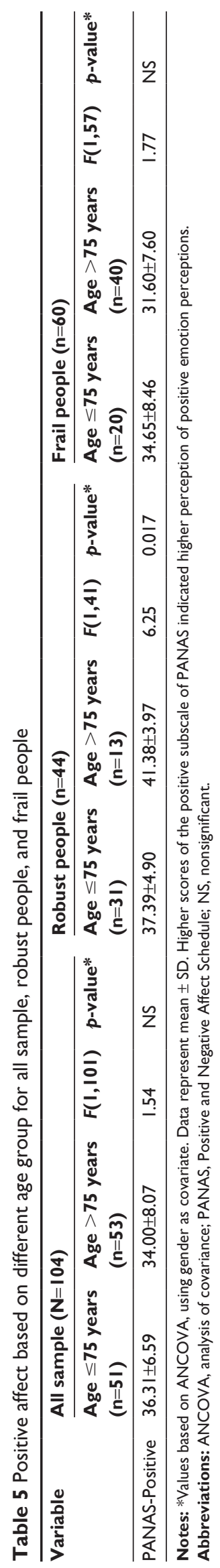

suppose that PA and NA may act indirectly on different individual behaviors. For example, affectivity can have a key role in the adoption and/or maintenance of a physically active lifestyle, ${ }^{37}$ development of creativity, ${ }^{38}$ execution of cognitive tasks, ${ }^{39}$ and in social experiences and interactions, ${ }^{40}$ which are recognized as considerable risk factors for the onset of frailty. Furthermore, PA is also associated with a reduction of neuroendocrine, inflammatory (eg, lower levels of cytokine Il-6), ${ }^{41}$ and cardiovascular activity. ${ }^{42}$ The obtained results may have two important implications. On the one hand, they make it possible to provide empirical evidence to the multidimensional theorization and definition of frailty. ${ }^{17,43} \mathrm{In}$ fact, it seems that a reduced level of PA and high level of NA may contribute to increases in the severity of frailty condition. In line with the view of frailty proposed by Gobbens et al in which psychological components are included, ${ }^{18}$ our results suggested that also emotion experience may be a useful construct to be taken into consideration in association with frailty condition. On the other hand, our findings may be a useful tool to increase the awareness of the important role of emotion experience in interventions for the prevention of frailty. For example, interventions of physical exercise or cognitive training associated with frequent experience of positive emotions may have a higher efficacy with respect to classical interventions.

Our results also demonstrated that robust individuals aged $\geq 76$ years significantly improved their PA compared to the group aged between 60 and 75 years, supporting the SST and the "positive effect" of aged people mentioned by Carstensen et al. ${ }^{14}$ On the contrary, no age differences were found in frail participants in terms of positive emotions. Our results on robust participants are consistent with those obtained by Carstensen et $\mathrm{al}^{3}$ and Gross et $\mathrm{al},{ }^{12}$ who noticed the most frequent presence of emotions such as happiness in seniors compared to young and adult people. The same tendency has also been observed in an Italian study conducted by Galati et al. ${ }^{44}$ However, these studies involved older adults who were not characterized as frail. Therefore, it is possible to assume that frailty may seriously compromise the health status of the individual, limiting the perception of PA, and the increase of PA with advancing age.

Results of this study should be interpreted in light of several limitations. First, the sample size was small with some subgroups (eg, robust older adults aged $\geq 76$ years, frail older adults aged $\leq 75$ years) comprising a very limited number of participants. Furthermore, the recruitment of participants was done in a limited area of Italy and was not based on randomization or stratification strategies, making it impossible to generalize the results to the entire Italian aged population. A further 
limitation concerns the cross-sectional design of this study, which did not allow for the analysis of trends of emotional experience and their impact on frailty condition. Also the use of self-report instruments with older adults could be seen as a study limitation, since self-reporting may be less accurate and more sensitive to cognitive impairment, level of education, and culture in comparison with performance-based assessment. ${ }^{45,46}$ Finally, the Italian version of the PANAS has been validated only on a sample of adult people. However, the original version of the instrument has been repeatedly used with older adults; ${ }^{27,47}$ therefore, it may be assumed that the Italian version preserves the same features as the original instrument, and consequently, it can be applied to aged people.

\section{Conclusion}

This study provides significant evidence on the association of both positive and negative emotion perception with frailty condition, giving new insights for the evaluation and prevention of frailty. Furthermore, results confirm the theory that proposes that positive emotions increases with the advancement of age in robust individuals. Due to these noteworthy limitations, our findings need to be deepened in the future with studies conducted on larger samples and longitudinally designed.

\section{Disclosure}

The authors report no conflicts of interest in this work.

\section{References}

1. Watson D, Clark LA, Tellegen A. Development and validation of brief measures of positive and negative affect: the PANAS scales. J Pers Soc Psychol. 1988;54(6):1063-1070.

2. Tellegen A. Structures of mood and personality and their relevance to assessing anxiety, with an emphasis on self-report. In: Tuma AH, Maser J, editors, Anxiety and the Anxiety Disorders. Erlbaum: Hillsdale; 1985:681-706.

3. Carstensen LL, Turan B, Scheibe S, et al. Emotional experience improves with age: evidence based on over 10 years of experience sampling. Psychol Aging. 2011;26(1):21-33.

4. Beiser M. Components and correlates of mental well-being. $J$ Health Soc Behav. 1974;15(4):320-327.

5. Clark LA, Watson D. Mood and the mundane: relations between daily life events and self-reported mood. J Pers Soc Psychol. 1988;54(2):296-308

6. Berges I-M, Seale GS, Ostir GV. The role of positive affect on social participation following stroke. Disabil Rehabil. 2012;34(25):2119-2123.

7. Fisher MN, Snih SA, Ostir GV, Goodwin JS. Positive affect and disability among older Mexican Americans with arthritis. Arthritis Rheum. 2004;51(1):34-39.

8. Ostir GV, Markides KS, Black SA, Goodwin JS. Emotional well-being predicts subsequent functional independence and survival. J Am Geriatr Soc. 2000;48(5):473-478.

9. Seale GS, Berges I-M, Ottenbacher KJ, Ostir GV. Change in positive emotion and recovery of functional status following stroke. Rehabil Psychol. 2010;55(1):33-39.

10. Clark L, Watson D. Diurnal variation in mood: interaction with daily events and personality. In: Meeting of the American Psychological Association; 1986; Washington, DC.
11. Carstensen LL. Evidence for a life-span theory of socioemotional selectivity. Curr Dir Psychol Sci. 1995;4(5):151-156.

12. Gross JJ, Carstensen LL, Pasupathi M, Tsai J, Götestam Skorpen C, Hsu AY. Emotion and aging: experience, expression, and control. Psychol Aging. 1997;12(4):590-599.

13. Charles S, Mather M, Carstensen L. Focusing on the positive: age differences in memory for positive, negative, and neutral stimuli. $J$ Exp Psychol. 2003;85:163-178.

14. Carstensen LL, Mikels JA. At the intersection of emotion and cognition aging and the positivity effect. Curr Dir Psychol Sci. 2005;14(3): $117-121$.

15. Roppolo M, Mulasso A, Gobbens RJ, Mosso CO, Rabaglietti E. A comparison between uni- and multidimensional frailty measures: prevalence, functional status, and relationships with disability. Clin Interv Aging. 2015;10:1669-1678.

16. Fried LP, Tangen CM, Walston J, et al. Frailty in older adults evidence for a phenotype. J Gerontol A Biol Sci Med Sci. 2001;56(3): M146-M157.

17. Campbell AJ, Buchner DM. Unstable disability and the fluctuations of frailty. Age Ageing. 1997;26(4):315-318.

18. Gobbens RJ, Luijkx KG, Wijnen-Sponselee MT, Schols JM. In search of an integral conceptual definition of frailty: opinions of experts. $J \mathrm{Am}$ Med Dir Assoc. 2010;11(5):338-343.

19. Markle-Reid M, Browne G. Conceptualizations of frailty in relation to older adults. $J$ Adv Nurs. 2003;44(1):58-68.

20. Roppolo M, Mulasso A, Rabaglietti E. Cognitive frailty in italian community-dwelling older adults: Prevalence rate and its association with disability. J Nutr Health Aging. 2017;21(6):631-636.

21. Ostir GV, Ottenbacher KJ, Markides KS. Onset of frailty in older adults and the protective role of positive affect. Psychol Aging. 2004;19(3): 402-408.

22. Park-Lee E, Fredman L, Hochberg M, Faulkner K. Positive affect and incidence of frailty in elderly women caregivers and noncaregivers: results of caregiver-study of osteoporotic fractures. $J$ Am Geriatr Soc. 2009;57(4):627-633.

23. [No authors listed]. Ethical principles of psychologists and code of conduct. Am Psychol. 2002;57(12):1060-1073.

24. Perneczky R, Wagenpfeil S, Komossa K, Grimmer T, Diehl J, Kurz A. Mapping scores onto stages: mini-mental state examination and clinical dementia rating. Am J Geriatr Psychiatry. 2006;14(2): 139-144.

25. Watson D, Tellegen A. Toward a consensual structure of mood. Psychol Bull. 1985;98(2):219-235.

26. Hilleras PK, Jorm AF, Herlitz A, Winblad B. Negative and positive affect among the very old: a survey on a sample age 90 years or older. Res Aging. 1998;20(5):593-610.

27. Kercher K. Assessing subjective well-being in the old-old the panas as a measure of orthogonal dimensions of positive and negative affect. Res Aging. 1992;14(2):131-168.

28. Terraciano A, McCrae RR, Costa PT Jr. Factorial and construct validity of the Italian Positive and Negative Affect Schedule (PANAS). Eur J Psychol Assess. 2003;19(2):131-141.

29. Garcia D, Kerekes N, Andersson Arntén A-C, Archer T. Temperament, character, and adolescents' depressive symptoms: focusing on affect. Depress Res Treat. 2012;2012:925372.

30. Norlander T, Bood S-Åk, Archer T. Performance during stress: affective personality, age, and regularity of physical exercise. Soc Behav Personal Int J. 2002;30(5):495-508.

31. Norlander T, Johansson $\AA$, Bood SÅ. The affective personality: its relation to quality of sleep, well-being and stress. Soc Behav Personal Int J. 2005;33(7):709-722.

32. Gobbens RJ, van Assen MA, Luijkx KG, Wijnen-Sponselee MT, Schols JM. The Tilburg frailty indicator: psychometric properties. J Am Med Dir Assoc. 2010;11(5):344-355.

33. Mulasso A, Roppolo M, Gobbens RJ, Rabaglietti E. The Italian version of the Tilburg Frailty Indicator: analysis of psychometric properties. Res Aging. 2016;38(8):842-863. 
34. Folstein MF, Folstein SE, McHugh PR. "Mini-mental state": a practical method for grading the cognitive state of patients for the clinician. J Psychiatr Res. 1975;12(3):189-198.

35. Nunnally J, Bernstein I. Psychometric Theory. 3rd ed. New York: McGraw-Hill; 1994

36. Mulasso A, Roppolo M, Giannotta F, Rabaglietti E. Associations of frailty and psychosocial factors with autonomy in daily activities: a cross-sectional study in Italian community-dwelling older adults. Clin Interv Aging. 2016;11:37-45.

37. Ostir GV, Cohen-Mansfield J, Leveille S, Volpato S, Guralnik JM. The association of positive and negative affect and exercise self-efficacy in older adults. J Aging Phys Act. 2003;11(2):265-274.

38. Filipowicz A. From positive affect to creativity: the surprising role of surprise. Creat Res J. 2006;18(2):141-152.

39. Ashby FG, Isen AM, Turken AU. A neuropsychological theory of positive affect and its influence on cognition. Psychol Rev. 1999;106(3): 529-550.

40. Berry DS, Hansen JS. Positive affect, negative affect, and social interaction. J Pers Soc Psychol. 1996;71(4):796-809.

41. Stellar JE, John-Henderson N, Anderson CL, Gordon AM, McNeil GD, Keltner D. Positive affect and markers of inflammation: discrete positive emotions predict lower levels of inflammatory cytokines. Emot Wash DC. 2015;15(2):129-133.
42. Steptoe A, Wardle J, Marmot M. Positive affect and health-related neuroendocrine, cardiovascular, and inflammatory processes. Proc Natl Acad Sci U S A. 2005;102(18):6508-6512.

43. Bergman H, Ferrucci L, Guralnik J, et al. Frailty: an emerging research and clinical paradigm - issues and controversies. J Gerontol A Biol Sci Med Sci. 2007;62(7):731-737.

44. Galati D, Sotgiu I, Lavagno G. Emozioni positive e vita quotidiana: uno studio su un gruppo di anziani. Delle Fave Cura Condivisione Beness Il Contrib Della Psicol Posit. 2007:94-109.

45. Nielsen LM, Kirkegaard H, Østergaard LG, Bovbjerg K, Breinholt K, Maribo T. Comparison of self-reported and performance-based measures of functional ability in elderly patients in an emergency department: implications for selection of clinical outcome measures. $B M C$ Geriatr. 2016;16(1):199.

46. Guralnik JM, Branch LG, Cummings SR, Curb JD. Physical performance measures in aging research. J Gerontol. 1989;44(5):M141-M146.

47. Mackinnon A, Jorm AF, Christensen H, Korten AE, Jacomb PA, Rodgers B. A short form of the positive and negative affect schedule: evaluation of factorial validity and invariance across demographic variables in a community sample. Personal Individ Differ. 1999;27(3): 405-416.
Clinical Interventions in Aging

\section{Publish your work in this journal}

Clinical Interventions in Aging is an international, peer-reviewed journal focusing on evidence-based reports on the value or lack thereof of treatments intended to prevent or delay the onset of maladaptive correlates of aging in human beings. This journal is indexed on PubMed Central, MedLine,

\section{Dovepress}

CAS, Scopus and the Elsevier Bibliographic databases. The manuscript management system is completely online and includes a very quick and fair peer-review system, which is all easy to use. Visit http://www.dovepress. com/testimonials.php to read real quotes from published authors. 